\title{
Glutathione ameliorates liver markers, oxidative stress and inflammatory indices in rats with renal ischemia reperfusion injury
}

\author{
Hassan Ahmadvand ${ }^{1,2}{ }^{\mathbb{D}}$, Esmaeel Babaeenezhad ${ }^{3,4^{*}}{ }^{\mathbb{D}}$, Maryam Nasri ${ }^{3}$, Leila Jafaripour ${ }^{5}$, Reza Mohammadrezaei \\ Khorramabadi $^{3}$
}

\author{
${ }^{1}$ Razi Herbal Medicine Research Center, Faculty of Medicine, Lorestan University of Medical Sciences, Khorramabad, Iran \\ ${ }^{2}$ Department of Biochemistry, Faculty of Medicine, Lorestan University of Medical Sciences, Khorramabad, Iran \\ ${ }^{3}$ Student Research Committee, Lorestan University of Medical Sciences, Khorramabad, Iran \\ ${ }^{4}$ Student of Veterinary Medicine, Faculty of Veterinary Medicine, Lorestan University, Khorramabad, Iran \\ ${ }^{5}$ Department of Anatomy, Faculty of Medicine, Dezful University of Medical Sciences, Dezful, Iran
}

\section{A R T I C L E I N F O}

\section{Article Type:}

Original

\section{Article History:}

Received: 10 February 2018

Accepted: 3 August 2018

Published online: 23 August 2018

\section{Keywords:}

Glutathione

Antioxidant enzymes

Myeloperoxidase

Renal ischemia reperfusion

\begin{abstract}
A B S T RAC T
Introduction: Glutathione (GSH) protects the tissue and cell from oxidative injury.

Objectives: In the current study, we investigated the possible effects of GSH on liver markers, oxidative stress and inflammatory indices in rat with renal ischemia reperfusion (RIR) injury. Materials and Methods: Twenty-four adult male Wistar rats were divided into 3 groups $(n=8)$. Group I (the control group), group II (the RIR group) received saline $(0.25 \mathrm{~mL} / \mathrm{d}$, intraperitoneally; i.p.), group III as the RIR group that received GSH $(100 \mathrm{mg} / \mathrm{kg} / \mathrm{d}$, i.p.). The treatment with saline or GSH began daily 14 days before RIR induction. RIR was induced by clamping renal pedicles for 45 minutes and 24 hours of reperfusion.

Results: RIR significantly increased the serum level of nitric oxide (NO), the serum activities of aspartate aminotransferase (AST), alkaline phosphatase (ALP), gamma-glutamyltransferase (GGT), the serum and renal levels of malondialdehyde (MDA), and the serum activity of myeloperoxidase (MPO). However, RIR significantly decreased the serum and renal levels of GSH, serum paraoxonase 1 (PON1) activity, and the serum and renal activities of catalase (CAT) and glutathione peroxidase (GPX). GSH administration could significantly improve the serum activities of AST, GGT, MPO, GPX and PON1 and serum levels of NO, renal MDA, GSH levels, and serum and also renal CAT activities.

Conclusion: Our study indicated that GSH administration ameliorated RIR injury in rats by improving the activities of liver markers and antioxidant enzymes, the levels of MDA, NO, GSH and MPO activity.
\end{abstract}

Implication for health policy/practice/research/medical education:

Our study indicated that glutathione could ameliorate lipid peroxidation, the activities of liver markers, antioxidant enzymes, and the levels of glutathione, nitric oxide, and myeloperoxidase activity in ischemia reperfusion injury treated group.

Please cite this paper as: Ahmadvand H, Babaeenezhad E, Nasri M, Jafaripour L, Mohammadrezaei Khorramabadi R. Glutathione ameliorates liver markers, oxidative stress and inflammatory indices in rats with renal ischemia reperfusion injury. J Renal Inj Prev. 2019;8(2):91-97. DOI: 10.15171/jrip.2019.18.

\section{Introduction}

Renal ischemia reperfusion (RIR) injury is one of the causes of acute kidney failure (AKF). It may be induced by some conditions such as sepsis, trauma, and kidney transplantation. Renal ischemia begins a series of incidents that can finally cause cell dysfunction and cell death. The restoration of blood to tissues, which underwent ischemia, can make the recovery of the normal tissue function, while the tissue injury is also induced by the reperfusion $(1,2)$. So far, the precise mechanisms of the pathogenesis of ischemia reperfusion (IR) injury have not been fully demonstrated. It has been suggested that multiple factors participate in the pathogenesis of IR injury such as hypoxia, free radical-mediated injury, inflammation, and cellular apoptosis. Acute ischemia modulates an acute inflammation which is demonstrated by neutrophil 
activation (2). Activated neutrophils make tissue damage through the generation and release of reactive oxygen metabolites and cytotoxic proteins such as myeloperoxidase (MPO), proteases, and lactoferrin into the extracellular space (3). Nevertheless, it is mainly believed that the principle factors which make kidney injury during IR are reactive oxygen species (ROS) and reactive nitrogen species (RNS) (4). Free radical overproduction after the reperfusion leads to cellular injury and the reduction of endogenous antioxidant enzyme activities (2). Hence, free radical scavengers can be helpful in RIR injury.

Glutathione (GSH) is considered as the main non-protein sulfhydryl compound that exists in the mammalian cells and protects the cell from oxidative injury. It contributes to many physiological processes. GSH is a free radical scavenger and an inhibiting lipid peroxidation (LPO). GSH is considered as a cofactor for some of antioxidant and detoxifying enzymes (5). The depletion of GSH, as a tripeptide, is associated with disorders such as aging diseases, cardiovascular diseases, cystic fibrosis, and neurodegenerative diseases (6). GSH plays a role in the regeneration of other antioxidants such as tocopherols and ascorbate (5).

\section{Objectives}

So far, the possible protective effects of GSH on liver markers, oxidative stress and inflammatory indices have not been investigated in RIR rats. Hence, this study aims to investigate the possible protective effects of GSH administration on liver markers, oxidative stress and inflammatory indices in rat with RIR injury.

\section{Materials and Methods}

\section{Animals and study design}

Twenty-four adult male Wistar rats (180-200 g) were purchased from the Tehran Pasteur Institute. The rats were housed in a room with stable conditions including the temperature of $22^{\circ} \mathrm{C}$, the humidity of $50 \pm 10 \%$, and the light-dark cycle of 12 hours at the animal lab of the Razi Herbal Medicines Research Center. One week was considered for animal acclimation to the new house. During the study, the rats were given enough food and water. The animals were randomly divided into three equal groups $(n=8)$. Group I as the control group, which did not undergo RIR; group II as the RIR group, which underwent $\mathrm{RIR}$ and received saline $(0.25 \mathrm{~mL} / \mathrm{d}$, intraperitoneally; i.p.); group III as the RIR group, which underwent RIR and received GSH (100 mg/kg/d, i.p.). All treatments (saline or GSH) began daily 14 days before RIR induction.

\section{Surgery procedure}

Under anesthesia condition with ketamine $(75 \mathrm{mg} / \mathrm{kg}$, i.p.) and xylazine ( $8 \mathrm{mg} / \mathrm{kg}$, i.p.), ischemia induction was performed. At first, the surface of abdomen was shaved and sterilized. Then, the abdomen was uncovered by the midline incision. After that, the right and left renal pedicles were detached carefully and clamped for 45 minutes by safe and none-traumatic clamps. After 45 minutes, the clamps were removed immediately to make reperfusion. Reperfusion period was considered 24 hours. During the surgery, humid and sterile gauze was used for the preservation of viscera. Additionally, warmed saline at the temperature of $37^{\circ} \mathrm{C}$ was splashed on viscera to keep moisture. The rats in the control group underwent RIR surgery procedure, as it was performed in the RIR group, but RIR was not induced.

\section{Biochemical study}

At the end of the reperfusion period, the animals were anesthetized with ketamine $(75 \mathrm{mg} / \mathrm{kg}$, i.p.) and xylazine (8 mg/kg, i.p.). Blood sampling was performed by blood collecting from their hearts. The samples were kept in the room temperature for 20 minutes in order to clot. Then, they were centrifuged immediately at $3000 \mathrm{rpm}$ for 15 minutes. After centrifugation, the serum samples were separated carefully and transmitted into microtubes. The serum samples were kept at $-70^{\circ} \mathrm{C}$ until the biochemical measurements. The right kidney was chosen for the measurement of renal biochemical parameters. It was removed and used fresh or kept frozen until the measurement of renal parameters.

\section{Determination of malondialdehyde levels}

In this study, serum and renal contents of malondialdehyde (MDA), as the marker of LPO, were evaluated according to the thiobarbituric acid (TBA) assay (7). The measurement of absorbance was performed spectrophotometrically at $532 \mu \mathrm{m}$. The concentrations of MDA were shown as nmol $\mathrm{MDA} / \mathrm{mg}$-pr.

\section{Determination of GSH levels}

Serum and renal level of GSH were determined in accordance with our previous study (8).

\section{Assessment of catalase activity}

Catalase (CAT) activity in serum and kidney was determined according to the Sinha method (9). The reaction was initiated by the addition of $20 \mu \mathrm{L}$ of the sample in $2 \mathrm{~mL}$ of $30 \mathrm{mmol} / \mathrm{L}$ hydrogen peroxide $(\mathrm{H} 2 \mathrm{O} 2)$ in $50 \mathrm{mmol} / \mathrm{L}$ potassium phosphate buffer ( $\mathrm{pH}$ 7.0). Enzyme units are considered as $\mathrm{mmol} / \mathrm{L}$ of consumed $\mathrm{H} 2 \mathrm{O} 2$ per min $\mathrm{g}$ or $\mathrm{mL}$.

\section{Assessment of GPX activity}

Serum and renal glutathione peroxidase (GPX) activities were determined in accordance with our previous study (10).

\section{Assessment of paraoxonase 1 activity}

To evaluate serum paraoxonase 1 (PON 1) activity, paraoxon was used as substrate. PON 1 activity was measured spectrophotometrically by increasing the absorbance at $412 \mathrm{~nm}$ due to the production of 4 -nitrophenol. The enzyme activity was measured at $25^{\circ} \mathrm{C}$ by the addition of 
$50 \mu \mathrm{L}$ of serum sample to Tris- $\mathrm{HCl}$ buffer $(1 \mathrm{~mL}, 100 \mathrm{mM}$ at $\mathrm{pH} 8.0$ ) including $2 \mathrm{mM} \mathrm{CaCl} 2$ and $5 \mathrm{mM}$ paraoxon. 4-nitrophenol production rate was measured at $412 \mathrm{~nm}$. Molar extinction coefficient of $17100 \mathrm{M}^{-1} \mathrm{~cm}^{-1}$ was used for the calculation of enzymatic activity (11).

\section{Assessment of myeloperoxidase activity}

The evaluation of serum myeloperoxidase (MPO) activity was done by a modified O-dianisidine method. For this evaluation, a mixture containing $0.3 \mathrm{~mL}$ of phosphate buffer $(0.1 \mathrm{M}, \mathrm{pH} 6.0), 0.3 \mathrm{~mL}$ of hydrogen peroxide $(0.01$ $\mathrm{M}), 0.5 \mathrm{~mL}$ of $\mathrm{O}$-dianisidine $(0.02 \mathrm{M})$ and the sample $(10$ $\mu \mathrm{L})$, was poured in a covet $(1 \mathrm{~cm}$ of path length). The final volume of the mixture was $3 \mathrm{~mL}$. The sample was added to the mixture in the end stage of mixture preparation and the changes of mixture absorbance in $460 \mathrm{~nm}$ were evaluated for 10 minutes. The activity of MPO was shown as unit/mg protein (12).

\section{Determination of NO levels}

The assay of serum nitric oxide (NO) concentration was done by the determination of nitrite which is the end product of NO. The measurement of nitrite was determined in accordance with our study (13).

\section{Ethical issues}

Our study protocols were approved by the Animal Ethics Committee of the Lorestan University of Medical Sciences. Also, they were in accordance with the guidelines of the National Health Institute (NIH1978) and Medical Research Council. Additionally, all experimental protocols and steps of the tests were conducted in compliance with the regulations of the Research Ethics Committee of Iranian Ethical Guidelines for the use of animals in research.

Statistical analysis

All results are indicated as mean \pm SD. The data comparison between groups was done by the LSD test. SPSS 13 for Windows was used for statistical analyses. A $P$ value of $<0.05$ was considered statistically significant.

Results

Effect of GSH on liver markers in RIR animals

The serum activities of liver markers including GGT, ALP, ALT, and AST are shown in Table 1. GGT activity in the untreated RIR group was significantly (2.49-fold) more than that of the control group. GSH significantly (51.10\%) decreased GGT activity in the treatment group compared to the untreated RIR group. The activity of ALP in untreated RIR animals was significantly (1.66-fold) higher than that of control rats. GSH (23.22\%) reduced ALP activity in the treatment group compared to the untreated RIR group. The decrease of ALP activity by GSH treatment was not statistically significant. The activity of ALT in untreated RIR rats was (1.59-fold) higher than that of control animals. GSH reduced (45.56\%) ALT activity in the treatment group compared to the RIR group, but the reduction of ALT activity was not statistically significant. The activity of AST in untreated RIR rats significantly (2.12-fold) increased compared to the control group. GSH significantly (35.39\%) reduced AST activity in the treatment group compared to the untreated RIR group.

\section{Effect of glutathione on serum and renal levels of MDA} in RIR animals

The levels of MDA in serum and kidney are respectively demonstrated in Tables 2 and 3. The significant increase (2.26-fold) of serum level of MDA was observed in untreated RIR rats in comparison to control rats. GSH could significantly (24.05\%) reduce serum MDA level in the treatment group compared to the untreated RIR group. The level of kidney MDA in untreated RIR rats was significantly (1.65-fold) higher than that of control animals. GSH significantly (32.08\%) inhibited the increase

Table 1. The effect of glutathione on activities of AST, ALT, ALP and GGT in the serum of control, untreated and treated RIR rats

\begin{tabular}{lccc}
\hline Parameter & Control & IR & IR+ glutathione \\
\hline AST (IU/L) & $102.38 \pm 8.82$ & $217.86 \pm 26.42 *$ & $140.75 \pm 15.95^{\#}$ \\
ALT (IU/L) & $198.75 \pm 43.82$ & $316.43 \pm 17.48$ & $172.25 \pm 69.14$ \\
ALP (IU/L) & $278.62 \pm 10.88$ & $463.71 \pm 65.17^{*}$ & $356.00 \pm 17.48$ \\
GGT (IU/L) & $1.27 \pm 0.16$ & $3.17 \pm 0.80^{*}$ & $1.55 \pm 0.13^{\#}$ \\
\hline
\end{tabular}

Data are shown as mean \pm SD. Significant difference compared with the control group is indicated with $* P<0.05$. Significant difference compared with the IR group is indicated with ${ }^{\#} P<0.05$.

Table 2. The effect of glutathione on levels of GSH, MDA, activities of GPX and CAT in the serum of control, untreated and treated RIR rats

\begin{tabular}{lccc}
\hline Experimental groups & GSH (U/mg protein) & MDA (nm/mg protein) & GPX (U/mg protein) \\
\hline Control & $12.86 \pm 0.37$ & $36.87 \pm 3.05$ & $3392.6 \pm 98.99$ \\
IR & $9.29 \pm 0.34^{*}$ & $83.50 \pm 5.75^{*}$ & $2072.7 \pm 141.37^{*}$ \\
IR + glutathione & $11.68 \pm 0.26^{* \#}$ & $63.41 \pm 4.85^{* \#}$ & $2899.5 \pm 106.59 * \#$ \\
\hline
\end{tabular}

Data are shown as mean \pm SD. Significant difference compared with the control group is indicated with $* P<0.05$. Significant difference compared with the IR group is indicated with ${ }^{\#} P<0.05$. 
Table 3. The effect of glutathione on levels of GSH, MDA, activities of GPX and CAT in the kidney of control, untreated and treated RIR rats

\begin{tabular}{lccc}
\hline Experimental groups & GSH (U/mg protein) & MDA (nm/mg protein) & GPX (U/mg protein) \\
\hline Control & $10.88 \pm 0.20$ & $22.63 \pm 2.19$ & $2749.0 \pm 282.41$ \\
IR & $5.28 \pm 0.27^{*}$ & $37.40 \pm 3.06^{*}$ & $1252.3 \pm 78.52^{*}$ \\
IR + glutathione & $12.12 \pm 0.45^{*}$ & $25.40 \pm 2.39^{*}$ & $1860.5 \pm 244.38^{*}$ \\
\hline
\end{tabular}

Data are shown as mean \pm SD. Significant difference compared with the control group is indicated with $* P<0.05$. Significant difference compared with the IR group is indicated with ${ }^{\#} P<0.05$.

of renal MDA in comparison to untreated RIR rats.

\section{Effect of glutathione on serum and kidney levels of GSH in RIR animals}

The levels of glutathione in serum and kidney are respectively shown in Tables 2 and 3. The level of serum glutathione in the untreated RIR group was significantly (1.38-fold) lower than that of control animals. GSH significantly $(25.72 \%)$ increased serum GSH level in the treatment group compared to the untreated RIR group. The level of kidney glutathione in untreated RIR rats was significantly (2.06-fold) lower than that of control rats. GSH significantly (129.54\%) increased the level of renal GSH in the treatment group compared to RIR rats.

\section{Effect of GSH on serum and renal CAT activities in RIR animals}

The activities of CAT in serum and kidney are respectively shown in Tables 2 and 3. The decrease of the serum CAT activity (2.14-fold) was significantly seen in RIR rats compared to control rats. Serum CAT activity significantly $(78.69 \%)$ increased in the treatment group by GSH administration compared to untreated RIR animals. The renal activity of CAT in the untreated RIR group was significantly (1.92-fold) lower than that of control rats. GSH administration in the treatment group significantly (79.62 \%) increased renal CAT activity in comparison with untreated animals.

\section{Effect of GSH on serum and renal GPX activities in RIR animals}

The activities of GPX in serum and kidney are respectively indicated in Tables 2 and 3. Serum GPX activity in untreated RIR rats was significantly (1.63-fold) lower than that of control animals. GSH could significantly (39.88\%) enhance serum GPX activity in the treatment group compared to untreated RIR animals. The activity of renal GPX in untreated RIR rats was significantly (2.19-fold) lower than that of control rats. GSH could enhance renal GPX activity (48.56\%) in the treatment group compared to untreated RIR animals, but it was not significant.

\section{Effect of GSH on serum PON 1 activity in RIR animals}

The PON 1 activity in serum is shown in Figure 1. The activity of serum PON 1 in untreated RIR rats was significantly (7.62-fold) lower than that of control rats. The treatment of RIR rats with GSH significantly
(448.26\%) increased serum PON 1 activity compared to the untreated RIR group.

\section{Effect of GSH on MPO activity in RIR animals}

The serum activity of MPO is shown in Figure 2. The activity of serum MPO in untreated RIR rats was significantly (4.40-fold) more than that of control animals. The treatment of RIR rats with GSH significantly (23.33\%) decreased serum MPO activity compared to untreated RIR animals.

\section{Effect of GSH on serum NO level in RIR animals}

The serum level of NO is shown in Figure 3. The level of serum NO in untreated RIR animals was significantly (1.21-fold) more than that of control animals. The

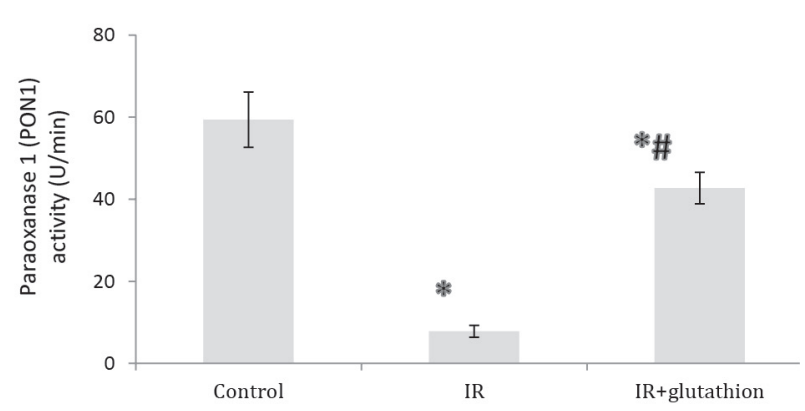

Figure 1. The effect of glutathione on serum paraoxonase 1 (PON 1) activity in control, untreated and treated RIR animals. Values are expressed as mean $\pm \mathrm{SD}$. *Shows significance of data comparing with the control group $(P<0.05)$. "Shows significance of data comparing with the IR group $(P<0.05)$.

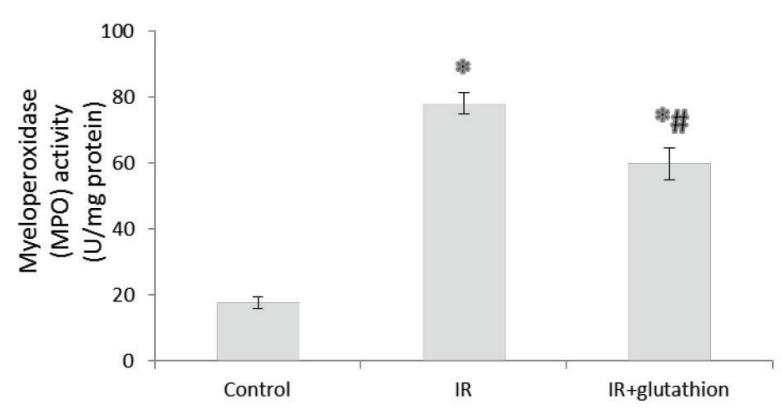

Figure 2. The effect of glutathione on serum myeloperoxidase (MPO) activity in control, untreated and treated RIR animals. Values are indicated as mean \pm SD. *Shows significance of data comparing with the control group $(P<0.05)$. "Shows significance of data comparing with the IR group $(P<0.05)$. 


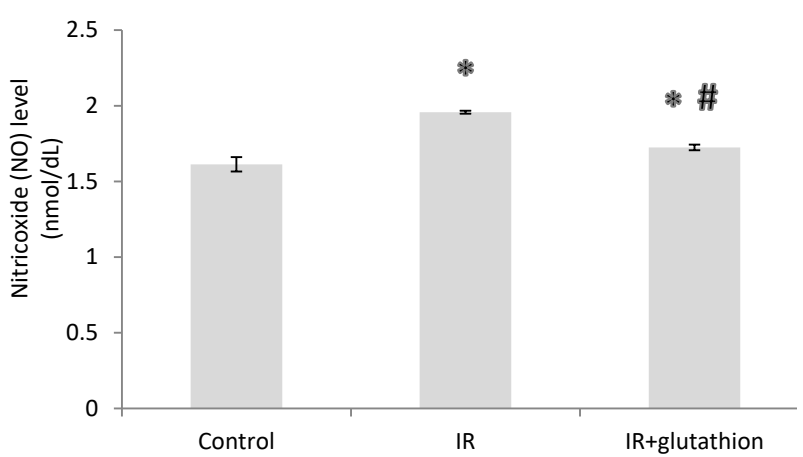

Figure 3. Effect of GSH on serum nitric oxide (NO) level in control, untreated and treated RIR animals. Values are expressed as mean $\pm \mathrm{SD}$. *Shows significance of data comparing with the control group $(P<0.05)$. \#Shows significance of data comparing with the IR group $(P<0.05)$

treatment of RIR rats with GSH could significantly (11.79\%) reduce NO level compared to the untreated RIR group.

\section{Discussion}

RIR is one of the events that lead to renal damage and the development of acute renal failure. It has been demonstrated that multiple factors participate in the pathogenesis of IR injury such as free radicals and inflammation $(1,2)$. Reactive oxygen or nitrogen species are the main elements which participate in RIR pathogenesis (4). Antioxidant enzymes such as superoxide dismutase (SOD) and CAT, and other antioxidants such as GSH preserve the cells against free radicals $(5,14)$. Hence, the administration of antioxidant is helpful for disorders which are associated with free radicals such as RIR.

The liver is a target organ that is undergone oxidative stress after RIR (15). The evaluation of liver enzymes is a nice procedure to examine liver function (16). In our study, the serum activities of liver markers including GGT, ALP, ALT, and AST increased in RIR rats compared to the control group, but the increase of ALT activity was not significant. These results showed that RIR induced hepatic damages as it was previously reported (15). All liver markers decreased after the treatment with GSH, but the reduction of ALP and ALT activities were not significant compared with the RIR group. GSH could decrease the activities of ALP and ALT in the treated group in a way that their activities were not significantly different from those in the control group $(P>0.05)$. Other reports showed that natural antioxidants could reduce the activities of liver markers and improve liver function in different pathologic states $(17,18)$. Another study showed that GSH administration could reduce the activity of liver markers in vivo (19). Our results are similar to other researches which were mentioned. Therefore, the use of natural antioxidants with hepatoprotective effect can be useful for ameliorating RIR-induced hepatic damage. The levels of serum and renal MDA significantly increased in the RIR group compared to control rats, while serum and kidney activities of CAT, GPX, GSH levels, and serum PON1 activity significantly decreased in the RIR group. The treatment with GSH could significantly ameliorate serum and renal MDA and GSH levels, the activities of serum and renal CAT, renal GPX, and serum PON1 in treated RIR rats compared with the untreated RIR group. Antioxidant enzymes and GSH are used for the investigation of oxidant/antioxidant status (20). It has been reported that natural antioxidants such as vitamin E (17), erythropoietin, melatonin (21), proanthocyanidin (18), and oleuropein (11) could decrease MDA level and increase the activities of antioxidant enzymes and GSH levels in pathological conditions induced by oxidative stress. Additionally, Chen et al noted the amelioration of oxidative stress and MDA level by GSH administration (19). The results of our study similar to the previous studies which were mentioned. Hence, the use of natural antioxidants such as GSH with advantageous effects on oxidative stress indices can either be helpful or prevent the complications which are associated with RIR-induced oxidative stress.

MPO is an enzyme that is related to oxidative stress and inflammation. It is located in the azurophilic granules of leukocytes including neutrophils and monocytes, and it is released in inflammatory conditions $(22,23)$. Our study showed that serum MPO activity significantly increased in the RIR group in comparison with control rats. Serum MPO activity reduced significantly in GSH treated RIR rats compared with the untreated RIR group. Many studies expressed that the natural antioxidants could decrease the MPO activity and suppress inflammation (23). Other results of our study indicated that RIR significantly increased NO level in the RIR group in comparison with control rats. The treatment of RIR rats with GSH could significantly reduce NO level compared to the untreated RIR group. It has been proposed that NO is generated by inducible NO synthase (iNOS) at high level in the proximal tubules as a response to ischemic injury. It has bad effects on kidney tissue during IR (2). Similar studies reported that natural antioxidants play a role in the reduction of NO level in vivo (24). Our results and other studies showed that natural antioxidants could reduce the level of NO and MPO activity. Hence, the consumption of natural antioxidants such as GSH with beneficial effects on NO and MPO can reduce the complications of RIR which are associated with oxidative stress and inflammation.

Our study and other researches showed that natural antioxidants exert beneficial effects on RIR complications. Therefore, antioxidant therapy can be considered as an important strategy to treat RIR complications in patients with RIR. Although the precise antioxidant and antiinflammatory mechanisms of GSH were not explained by our study, other studies showed these detailed mechanisms.

1. GSH directly scavenges free radicals such as hydroxyl 
radicals, singlet oxygen, and superoxide onions. It gives an electron to $\mathrm{H} 2 \mathrm{O} 2$ to reduce it and produce $\mathrm{H} 2 \mathrm{O}$ and $\mathrm{O} 2$. GSH has a major role in the regeneration of other antioxidants such as tocopherols and ascorbate $(5,25)$. 2. GSH gives protons to lipids' membrane in the cell and preserves them from oxidant agents (26). It also inhibits nuclear LPO by the increase of a-tocopherol capability (27).

3. GSH decreases the expression of inducible NO synthase (iNOS) protein and NO level in vivo (28). It also inhibits the production of peroxynitrite, as a cellular oxidant agent, through the elimination of ROS by GSH peroxidase (29).

4. GSH suppresses inflammation through p38MAPK signal pathway in vivo. It reduces the protein expression of cyclooxygenase-2 (COX-2) and the phosphorylation of p38 (28). Thus, our results seem to be reasonable.

\section{Conclusion}

Our study revealed that GSH caused protective effects against RIR in rats by the amelioration of liver function parameters, the activity of antioxidant enzymes, LPO, NO, and MPO activity. Hence, GSH as an antioxidant that has many advantageous effects can be suggested to patients with RIR complications. Protective effects on the liver function, antioxidant status, and inflammatory markers can ameliorate RIR-induced complications.

\section{Acknowledgments}

The authors thank the Lorestan University of Medical Sciences, Khorramabad, Iran. They also thank the chairman and staffs of the Razi Herbal Medicines Research Center of the Lorestan Medical University for their financial support of our research.

\section{Authors' contribution}

HA designed the project. EB, MN, LJ and RMK; collected the data. HA analyzed the data. EB wrote the manuscript. HA revised the English version and edited the final draft. All authors signed the manuscript.

\section{Conflicts of interest}

The authors declare that they have no conflicts of interest.

\section{Ethical considerations}

Ethical issues (including plagiarism, data fabrication, double publication) have been completely observed by the authors.

\section{Finding/Support}

The source of funding was provided by the Lorestan University of Medical Sciences.

\section{References}

1. Koo DD, Welsh KI, West NE, Channon KM, Penington AJ, Roake JA, et al. Endothelial cell protection against ischemia/reperfusion injury by lecithinized superoxide dismutase. Kidney Int. 2001;60:786-96. doi: 10.1046/j.1523- 1755.2001.060002786.x.

2. Malek M, Nematbakhsh M. Renal ischemia/reperfusion injury; from pathophysiology to treatment. J Renal Inj Prev. 2015;4:20-7. doi: 10.12861/jrip.2015.06.

3. Matthijsen RA, Huugen D, Hoebers NT, De Vries B, PeutzKootstra CJ, Aratani Y, et al. Myeloperoxidase is critically involved in the induction of organ damage after renal ischemia reperfusion. Am J Pathol. 2007;171:1743-52. doi: 10.2353/ajpath.2007.070184.

4. Devarajan P. Update on mechanisms of ischemic acute kidney injury. J Am Soc Nephrol. 2006;17:1503-20. doi: 10.1681/ASN.2006010017.

5. Schmitt B, Vicenzi M, Garrel C, Denis FM. Effects of $\mathrm{N}$-acetylcysteine, oral glutathione (GSH) and a novel sublingual form of GSH on oxidative stress markers: A comparative crossover study. Redox Biol. 2015;6:198-205. doi: 10.1016/j.redox.2015.07.012.

6. Ballatori N, Krance SM, Notenboom S, Shi S, Tieu $\mathrm{K}$, Hammond CL. Glutathione dysregulation and the etiology and progression of human diseases. Biol Chem. 2009;390:191-214. doi: 10.1515/BC.2009.033.

7. Ahmadvand H, Bagheri S, Khosrobeigi A, Boshtam M, Abdolahpour F. Effects of olive leaves extract on LDL oxidation induced-CuSO(4) in vitro. Pak J Pharm Sci. 2012;25:571-5.

8. Pourkhodadad S, Alirezaei M, Moghaddasi M, Ahmadvand H, Karami M, Delfan B, et al. Neuroprotective effects of oleuropein against cognitive dysfunction induced by colchicine in hippocampal CA1 area in rats. J Physiol Sci. 2016;66:397-405. doi: 10.1007/s12576-016-0437-4.

9. Sinha AK. Colorimetric assay of catalase. Anal Biochem. 1972;47:389-94. doi: 10.1016/0003-2697:90132-7.

10. Ahmadvand H, Ghasemi Dehnoo M, Cheraghi R, Rasoulian B, Ezatpour B, Azadpour M, et al. Amelioration of altered serum, liver, and kidney antioxidant enzymes activities by sodium selenite in alloxan-induced diabetic rats. Rep Biochem Mol Biol. 2014;3:14-20.

11. Ahmadvand H, Bagheri S, Tamjidi-Poor A, Cheraghi M, Azadpour M, Ezatpour B, et al. Effects of oleuropein on lipid peroxidation, lipid profile, atherogenic indices, and paraoxonase 1 status in gentamicin-induced nephrotoxicity in rats. ARYA Atheroscler. 2016;12:87-93.

12. Khalatbary AR, Ahmadvand H. Effect of oleuropein on tissue myeloperoxidase activity in experimental spinal cord trauma. Iran Biomed J. 2011;15:164-7. doi: 10.6091/ IBJ.1026.2012.

13. Ahmadvand $\mathrm{H}$, Shahsavari G, Tavafi M, Bagheri S, Moradkhani MR, Kkorramabadi RM, et al. Protective effects of oleuropein against renal injury oxidative damage in alloxan-induced diabetic rats; a histological and biochemical study. J Nephropathol. 2017;6:204-209. doi: 10.15171/jnp.2017.34.

14. Cornejo-Garcia J, Mayorga C, Torres M, Fernandez T, R-Pena R, Bravo I, et al. Anti-oxidant enzyme activities and expression and oxidative damage in patients with non-immediate reactions to drugs. Clin Exp Immunol. 2006;145:287-95. doi: 10.1111/j.1365-2249.2006.03149.x.

15. Khastar H, Kadkhodaee M, Sadeghipour Hr, Seifi B, Hadjati J, Najafi A, et al. Liver oxidative stress after renal ischemiareperfusion injury is leukocyte dependent in inbred mice. Iran J Basic Med Sci. 2011;14:534-9. doi: 10.22038/ ijbms.2011.5052.

16. Arora R, Bhushan S, Kumar R, Mannan R, Kaur P, Singh AP, 
et al. Hepatic dysfunction induced by 7, 12-dimethylbenz (a) anthracene and its obviation with erucin using enzymatic and histological changes as indicators. PLoS One. 2014; 9:e112614. doi: 10.1371/journal. pone.0112614.

17. Khastar H. Protective effects of vitamin E against liver damage caused by renal ischemia reperfusion. Ren Fail. 2015;37:494-6. doi:10.3109/0886022X.2015.1006084.

18. Mansouri E, Khorsandi L, Abedi HA. Antioxidant effects of proanthocyanidin from grape seed on hepatic tissue injury in diabetic rats. Iran J Basic Med Sci. 2014;17:460-4. doi: 10.22038/IJBMS.2014.2932.

19. Chen J, Wu F, Long Y, Yu W. Glutathione supplementation attenuates oxidative stress and improves vascular hyporesponsiveness in experimental obstructive jaundice. Oxid Med Cell Longev. 2015;2015: 486148. doi: $10.1155 / 2015 / 486148$.

20. Flores-Mateo G, Carrillo-Santisteve P, Elosua R, Guallar E, Marrugat J, Bleys J, et al. Antioxidant enzyme activity and coronary heart disease: meta-analyses of observational studies. Am J Epidemiol. 2009;170:135-47. doi: 10.1093/aje/ kwp112.

21. Ahmadiasl N, Banaei S, Alihemmati A. Combination antioxidant effect of erythropoietin and melatonin on renal ischemia-reperfusion injury in rats. Iran J Basic Med Sci. 2013;16:1209-16. doi: 10.22038/ijbms.2013.1978.

22. Klebanoff SJ. Myeloperoxidase: friend and foe. J Leukoc Biol. 2005;77:598-625. doi: 10.1189/jlb.1204697.

23. Han J, Ma D, Zhang M, Yang X, Tan D. Natural antioxidant betanin protects rats from paraquat-induced acute lung injury interstitial pneumonia. BioMed Res Int. 2015;2015. doi: $10.1155 / 2015 / 608174$.

24. Ahmadvand H, Shahsavari G, Tavafi M, Bagheri S, Moradkhani MR, Kkorramabadi RM, et al. Protective effects of oleuropein against renal injury oxidative damage in alloxan-induced diabetic rats; a histological and biochemical study. J Nephropathol. 2017;6:204-9. doi: 10.15171/jnp.2017.34.

25. El-Beltagi HS, Mohamed HI. Reactive oxygen species, lipid peroxidation and antioxidative defense mechanism. Not Bot Horti Agrobot Cluj-Napoca. 2013;41:44-57. doi: $10.15835 /$ nbha4118929.

26. Birben E, Sahiner UM, Sackesen C, Erzurum S, Kalayci O. Oxidative stress and antioxidant defense. World Allergy Organ J. 2012;5:9-19. doi:10.1097/ WOX.0b013e3182439613.

27. Tirmenstein M, Reed DJ. Effects of glutathione on the alpha-tocopherol-dependent inhibition of nuclear lipid peroxidation. J Lipid Res. 1989;30:959-65.

28. Sun S, Zhang H, Xue B, Wu Y, Wang J, Yin Z, et al. Protective effect of glutathione against lipopolysaccharideinduced inflammation and mortality in rats. Inflamm Res. 2006;55(11):504-10.

29. Sies H, Sharov VS, Klotz L-O, Briviba K. Glutathione peroxidase protects against peroxynitrite-mediated oxidations a new function for selenoproteins as peroxynitrite reductase. J Biol Chem. 1997;272:27812-7. doi: $10.1074 /$ jbc.272.44.27812.

Copyright @ 2019 The Author(s); Published by Nickan Research Institute. This is an open-access article distributed under the terms of the Creative Commons Attribution License (http://creativecommons.org/licenses/by/4.0), which permits unrestricted use, distribution, and reproduction in any medium, provided the original work is properly cited. 